\title{
Antiproton Physics Beyond the Low-Energy Frontier
}

\author{
J. Eades \\ CERN, Geneva, Switzerland
}

\begin{abstract}
Pioneering studies are now underway of low energy antiprotons below the present 1 Mev frontier to the lowest energies normally studied with particles of ordinary matter labout 1 $\mu \mathrm{eV})$. Paradoxically, some of the results of this work may shed light on physics at Big Bang energies of $10^{19} \mathrm{GeV}$. Many of the novel experimental techniques are drawing on particle trapping methods pioneered by the 1989 Nobel laureates.
\end{abstract}

Mention of the frontier of particle physics usually calls to mind work done with the highest energy particles available. More logically, however, a frontier area of physics is any domain in which we have little empirical knowledge. It is in this sense that the study of very low energy phenomena involving antiprotons is a true frontier.

The reasons for this apparent paradox are as follows. Antiprotons are produced by directing a proton beam onto a metallic target. The mass energy necessary to create the $\bar{p}$ is then provided by $\mathrm{p}$-nucleon collisions in the target nuclei. However, baryon conservation requires the creation of an extra nucleon (baryon number $=+1$ ) to compensate for the antiproton (baryon number = $-1)$. We must accelerate the proton beam to $5.6 \mathrm{GeV}$ to create all this extra mass-energy. Just at this threshold, the four particles (two old, two new) will be at rest in the centre of mass coordinate system, but the Lorentz transformation associated with $5.6 \mathrm{GeV}$ of beam energy will boost them to about $1 \mathrm{GeV}$ in the laboratory. Moreover, few antiprotons are produced just at threshold. If we want large numbers of $\bar{p}$, we must go well above the threshold energy, thereby boosting the (laboratory) energy still

Dr. John Eades has been an experimentalist with CERN, CH-1211 Geneva, Switzerland for 20 years. A graduate of Liverpool University, he has worked at Columbia University, NY and the Niels Bohr Institute, Copenhagen. $\mathrm{He}$ is interested in the physics of ultra-low energy protons and the ring imaging technique for Cerenkov radiation. higher. If we ask not only for large numbers, but large numbers at low energy we need a decelerator as well as the original accelerator. This argument ignores many important details but it easy to see why, in the years following the discovery of the antiproton in 1955 , low energy processes in $\bar{p}$ physics were difficult to study well.

The advent of the LEAR machine in 1984 changed this situation dramatically. LEAR produces high quality, low energy $\bar{p}$ beams by taking particles from CERN's antiproton storage complex at $3.5 \mathrm{GeV} / \mathrm{c}$, decelerating them in a ring of magnets, and cooling away the unavoidable (Liouville theorem) phase space blow-up [see Europhys. News 15 (July 1984)]. The LEAR operations group has now pushed the frontier down to $5 \mathrm{MeV}$, and $2 \mathrm{MeV}$ beams with sharply defined energy and a few $\mathrm{mm}^{2}$ cross-sectional area should soon be routinely available. The velocity of a $2 \mathrm{MeV} \overline{\mathrm{p}}$ is still about $2 \times 10^{7} \mathrm{~m} / \mathrm{s}$, so that in precision low energy antiproton work we are just entering the nonrelativistic domain (from above).

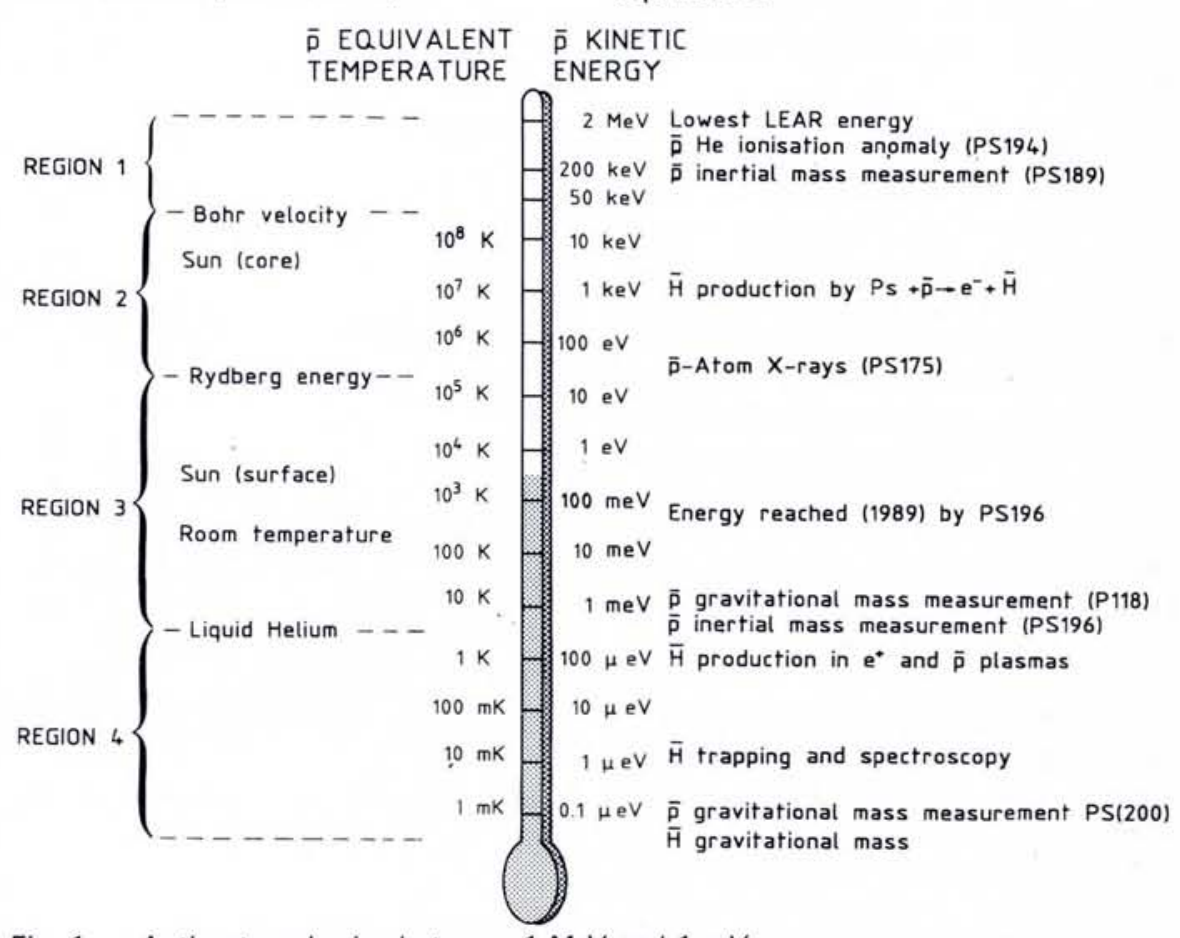

Fig. 1 - Antiproton physics between $1 \mathrm{MeV}$ and $1 \mu \mathrm{eV}$.

\section{From $\mathrm{MeV}$ To $\mu \mathrm{eV}$}

My purpose here is to review the scope and potential of antiproton physics between about $1 \mathrm{MeV}$ and $1 \mu \mathrm{eV}$ - from the current frontier to the lowest energy normally studied with ordinary particles of matter. An instructive way of surveying this field is the logarithmic 'antiproton thermometer' of Fig 1. Nature has provided some useful signposts to subdivide this enormous domain into several smaller ones:

Region 1: $\overline{\mathrm{p}}$ velocity above the Bohr velocity $\alpha c(\alpha=1 / 137$, the fine structure constant). The Bohr velocity is that of a ground state hydrogen electron; the corresponding $\overline{\mathrm{p}}$ energy is $25 \mathrm{keV}$.

Region $2: \overline{\mathrm{p}}$ velocity below $\alpha c, \overline{\mathrm{p}}$ energy above the Rydberg energy $E_{\mathrm{R}}(13.6 \mathrm{eV})$. $E_{\mathrm{R}}$ is the binding energy of a ground state hydrogen electron (and also its KE, $\left.1 / 2 m_{e}(\alpha c)^{2}\right)$.

Region 3: $\overline{\mathrm{p}}$ energy between $E_{\mathrm{R}}$ and $0.36 \mathrm{meV}$. The temperature equivalent of $E_{\mathrm{R}}$ is $1.6 \times 10^{5} \mathrm{~K}$ (taking $E \sim k T$ ), $0.36 \mathrm{meV}$ corresponds to $4.2 \mathrm{~K}$.

Region 4: $\overline{\mathrm{p}}$ energy below the $4.2 \mathrm{~K}$ equivalent. 
As we approach and pass these critical energies, we must expect that the character of the technical problems encountered will change as well as the nature of the physical phenomena of interest. We know for example that the Bethe-Bloch energy loss formula becomes inadequate for projectiles near the Bohr velocity and that atomic capture (and subsequent annihilation) of the antiproton will become important near the Rydberg energy. That gravitational effects may just be measurable in region 4 is hinted at by the fact that an antiproton dropped (in vacuo!) from the Jungfraujoch observatory should have the energy equivalent to $4.2 \mathrm{~K}$ when it reaches sea level. Galileo's 'high tower in Pisa' would only accelerate it to $\mathbf{5 . 5}$ $\mu \mathrm{eV}(64 \mathrm{mK})$.

\section{Recent Results}

\section{Region 1}

Work below a few $\mathrm{MeV}$ is now done by degradation of higher energy beams in absorbers (as was the case in preLEAR days below about $1 \mathrm{GeV}$ ). Several surprises have been revealed in this way. In region 1 for example, an Åarhus/CERN/PSI/Stockholm collaboration (experiment PS 194) studying $\overline{\mathrm{p}}$ atom collisions between $4 \mathrm{MeV}$ and 50 $\mathrm{keV}$ has found that the double ionisation cross-section in $\mathrm{He}$ is considerably larger for antiprotons than it is for protons ([1], Fig. 2). Theoretical attempts have been made to explain this in terms of interference effects between different double ionising processes, but the phenomenon is still not well understood. The mystery is further compounded by similar double ionisation results on $\mathrm{Ne}$ and $\mathrm{Ar}$ and triple ionisation effects on $\mathrm{Ne}$ only.

\section{Region 2}

At the lower end of region 2, much success has been achieved in antiprotonic atom work by L.M. Simons et al. (PS175-CERN/Jülich/Karlsruhe/PSI). A degradation technique in gases at about 10 mbar pressure is used to reach the vicinity of $E_{\mathrm{R}}$ where capture takes place to an initial state with principal quantum number $n$ of order 40. Among the many interesting results 1 mention only two. The first is the demonstration of complete removal of electrons from the heavy noble gases $\mathrm{Ne}, \mathrm{Ar}$, and $\mathrm{Kr}$ by the captured antiproton, with similar but less well established results in $\mathrm{Xe}$ [2]. The stripping proceeds in onion peeling fashion, electron shells being successively discarded as the antiproton cascades towards its ground state. Were the antiproton in orbit around a bare nucleus, it would de-excite itself below

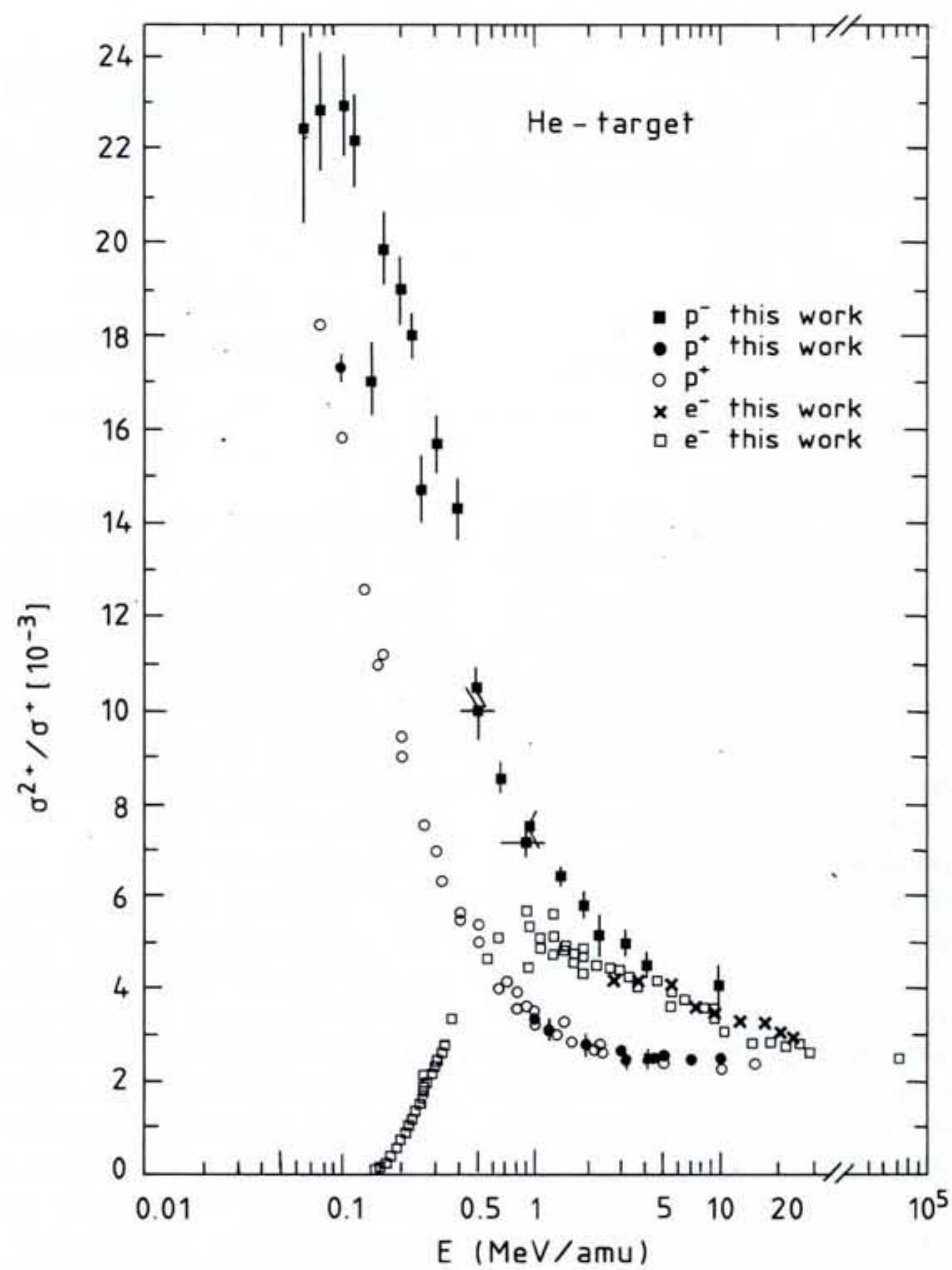

Fig. 2 - Ratio of double to single ionisation cross-sections in $\bar{H}$ for $p$ and $\bar{p}$. Energies have been divided by the projectile mass in atomic mass units to permit comparison with $e^{+}$ and $e^{-}$data at the same velocity.

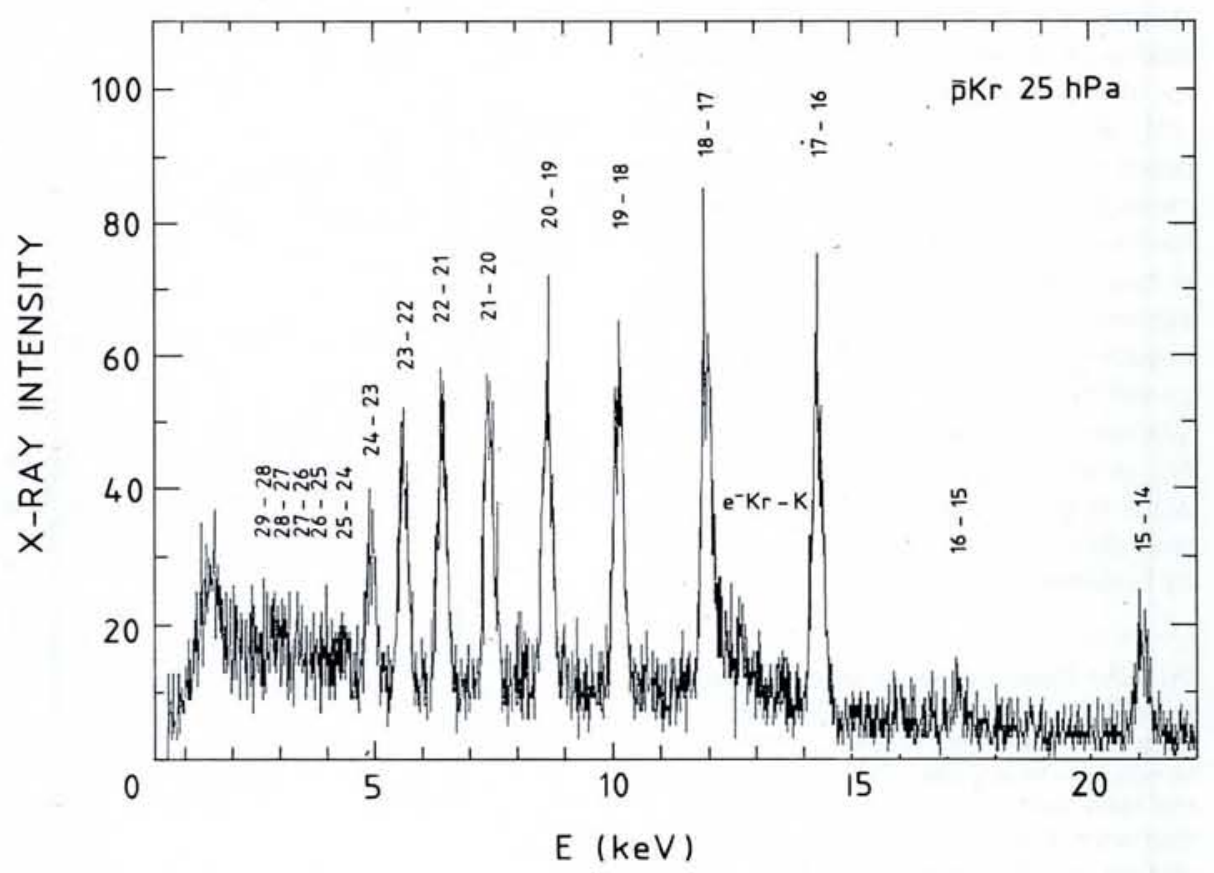

Fig. 3 - X-ray intensities (arbitrary units) from antiprotonic krypton. 
$n \sim 30$ by a sequence of $n \rightarrow n-1$ transitions with steadily increasing energy, each transition yielding one $\mathrm{X}$-ray in the $\mathrm{keV}$ region. In reality the atom is 'clothed' and the dominant process is Auger ejection of electrons by internal absorption of the $\mathrm{X}$-rays. The effect is exhibited for $\mathrm{Kr}$ in Fig. 3. The $29 \rightarrow 28$, $28 \rightarrow 27, \ldots 25 \rightarrow 24$ lines are suppressed because the $\mathrm{X}$-rays eject $\mathrm{L}$-, $\mathrm{M}$ and $\mathrm{N}$-shell electrons. When these electrons have all gone, the radiative cascade appears (transitions $24 \rightarrow 23$ down to $17 \rightarrow 16$ ) only to disappear again when the $\mathrm{X}$-ray energy is sufficient to deplete the K-shell $(16 \rightarrow 15$ and $15 \rightarrow 14)$. The proof that all L-, M- and $\mathrm{N}$-electrons have gone is that the sequence which follows has (when corrected for detector efficiency) the equal-yield characteristic of a pure radiative mechanism.

The second result concerns the protonium $(\mathrm{p} \overline{\mathrm{p}})$ atom. This has many similarities to the hydrogen atom, but since the radius of Bohr orbits is inversely proportional to the reduced mass of the orbiting particle, protonium is physically much smaller than hydrogen. The antiproton wave-function therefore penetrates the nuclear field region at low- $n$ atomic levels and the quantum states are shifted and broadened in energy relative to the Coulomb field values. Recent values [3] for the ground state shift $\Delta E$ and width $\Gamma$ obtained by observing the $\mathrm{K}_{\alpha}(2 \mathrm{P} \rightarrow 1 \mathrm{~S})$ transition are:

$$
\begin{aligned}
\Delta E & =-731 \pm 22 \mathrm{eV} \\
\Gamma & =1179 \pm 80 \mathrm{eV}
\end{aligned}
$$

These observables are simply related to the $\bar{p}$ p scattering length which is important in choosing between nucleonantinucleon strong interaction potential models.

\section{Region 3}

Work in region 3 implies the use of much lower pressures since capture is now an effect to be avoided rather than studied. The antiprotons must also be prevented by electromagnetic constraints from annihilating on the walls of their container. A Harvard/Mainz/ Washington group (PS196) aims to make a precision measurement of the antiproton inertial mass by studying antiprotons in just such a confining field. Antiprotons are first degraded in a foil to a few keV and loaded into a 'trap' formed by superposing a uniform $6 \mathrm{~T}$ magnetic field on a quadrupolar electrostatic well (Fig. 4). The trap is also loaded with the order of 2000 electrons (the ratio $m_{\mathrm{p}} / m_{\mathrm{e}}$ ) per antiproton. Kinetic energy can then be transferred from the antiproton cloud to the electron cloud.
The rate at which the electrons dissipate this energy by synchrotron radiation is much higher than the rate at which they receive it. Continuous cooling of the antiprotons with a time constant of a few seconds is thus observed to an energy below $100 \mathrm{meV}$, at which point the electrons are resonantly expelled. The remaining small cloud of cold antiprotons and the electromagnetic field together form a bound state analogous to a many-electron atom. The response to RF excitation of this 'geonium atom' (the term is due to H.G. Dehmelt, the 1989 Nobel laureate) then determines the antiproton inertial mass to extremely high precision. Preliminary results from a cloud of a few hundred $\bar{p}$ give a value equal to the proton mass to a few parts in $10^{7}$. However, just as the metrological value of the hydrogen atom is superior to that of a many-electron atom, so the ultimate precision of the geonium method is only reached when a single $\bar{p}$ occupies the trap. Work is in progress to reduce the number of trapped antiprotons to one, thereby reaching a precision of one part in $10^{9}$ (present accuracy is one part in $10^{5}$ ).

\section{Improved Deceleration Techniques}

Degrading foils make imperfect decelerators since they introduce multiple scattering and straggling, implying reduced beam quality (increased 'emittance') and large $\bar{p}$ losses. In addition, neither the environment of the $\bar{p}$ after deceleration nor the processes then to be studied are well controlled.

For experiments requiring antiprotons at a specific energy a Radio Frequency Quadrupole (RFQ) provides a way to avoid all these inconveniences. It consists of four parallel electrodes placed symmetrically about the beam axis. These are so shaped that their separation varies periodically along the beam direction. When the electrodes are excited with RF power, successive RF halfcycles produce the alternate focussing and defocussing forces necessary to prevent divergence of any finite emittance beam, while the periodic electrode separation produces a longitudinal decelerating force.

For more general purpose deceleration from a few MeV to a few Rydbergs a CERN/Budapest/Genova/Heidelberg/ Pisa/PSI collaboration (proposal P118) has suggested a modification of the cyclotron trap method used to obtain the results on electron stripping and strong interaction shifts cited above. In this device, the beam is injected at a few $\mathrm{MeV}$ into the outer part of a cyclotronlike field. Deceleration is not achieved by an RF field but by degradation in low pressure gas introduced into the space between the magnet poles. If the gas pressure is low enough the emittance increase is counteracted by the well known focussing properties of cyclotron fields. The $\bar{p}$ thus spiral down to form a trapped cylindrical swarm (volume several $\mathrm{cm}^{3}$ ) at the magnet centre. In the work described above, the gas whose properties were being studied served both as the decelerator and the capture target. The new feature is to reduce the pressure by a few orders of magnitude, allowing time to remove the $\bar{p}$ from the gas before capture can take place. The 'cyclotron trap' is no longer a trap but a complete 'anticyclo-

Fig. 4 - Typical electrostatic antiproton trap used by experiment PS196 in a 6 T magnetic field. Compare this 'tabletop' apparatus with the LEP detectors shown in Europhys. News 20 (June 1989).

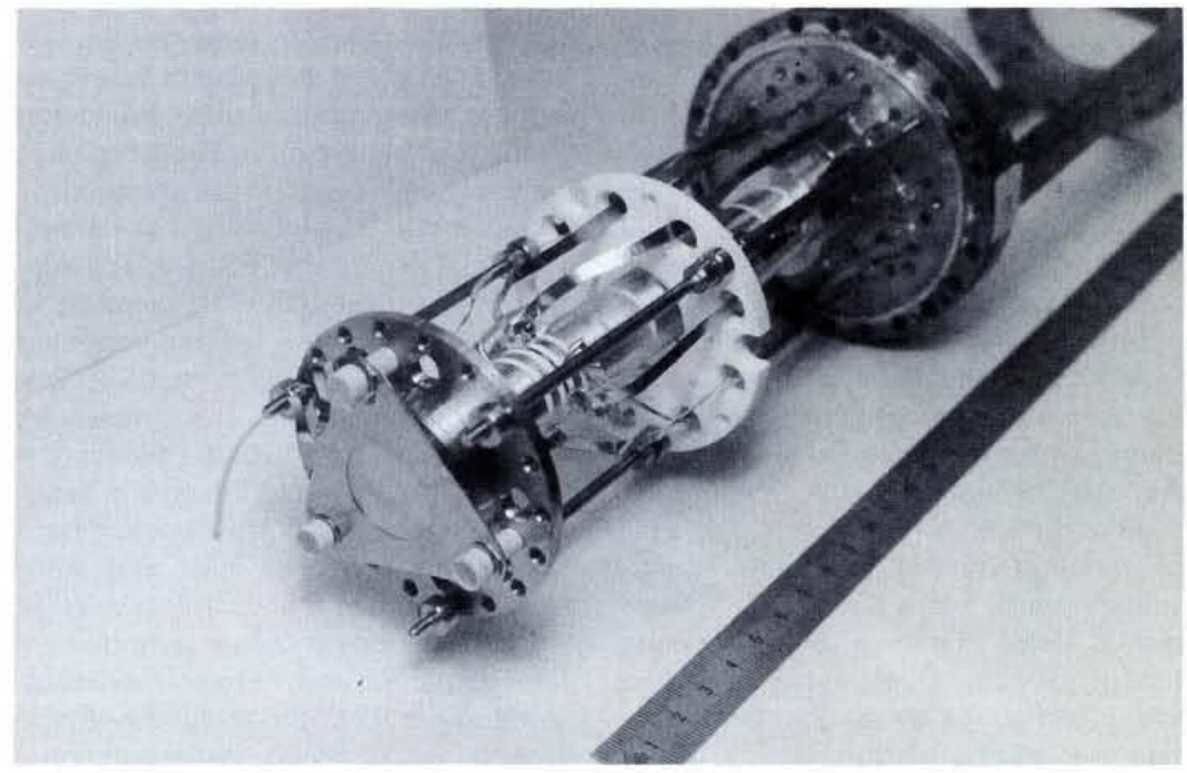


tron' with extraction of the $\bar{p}$ swarm through a hole in the pole face [4]. This will be achieved by an electric field of about $0.5 \mathrm{kV} / \mathrm{cm}$ which will be switched on when the mean swarm energy reaches a few keV and will reaccelerate the $\bar{p}$ through a very thin foil into a region of high vacuum ( $<10^{-13} \mathrm{mbar}$ ). Here the $\bar{p}$ will be confined in a trap similar to that shown in Fig. 4 where further cooling/deceleration will take place to the vicinity of $E_{\mathrm{R}}$ using a stochastic technique developed in Genova. The advantages offered are an increase of lifetime against capture from a few $\mu$ s to many hours and a controlled, more accessible environment for subsequent study of the $\bar{p}$ sample. The same collaboration has suggested additional cooling stages to reach $4.2 \mathrm{~K}$.

\section{Future Experiments}

Region 1

With these new and old tools, numerous adventurous experiments in all four energy regions are under preparation or discussion [see Hyperfine Interactions 44 (1989) 1-4]. The interaction of ionising radiation with matter is the basis of most high energy physics techniques, and a general study of antiproton ionisation phenomena in region 1 including extended measurements on multiple ionisation will be of immense value. Also in this region, and reflecting the importance of precise checks of CPT theorem predictions, an independent $\mathrm{p} / \mathrm{p}$ inertial mass comparison is in preparation (PS189-CERN/Orsay). An RFQ will be used to decelerate antiprotons from $2 \mathrm{MeV}$ to $200 \mathrm{keV}$. The particles will then be introduced into a $1 \mathrm{~m}$ diameter mass spectrometer magnet, where their cyclotron frequency will be compared with that of $\mathrm{H}^{-}$ions. The expected precision on the $\bar{p} / \mathrm{p}$ mass difference is again a few parts in $10^{9}$ with first results expected at the end of 1990.

\section{Region 2}

In region 2, vastly improved exotic atom studies and QED studies of highly ionised atoms will form the initial research program of $\mathrm{P} 118$ with the anticyclotron. Also at these energies, the feasibility of synthesising an antihydrogen beam for spectroscopic studies is being enthusiastically pursued by an Åarhus/University College, London/Los Alamos group. Antihydrogen, the simplest bound state of antimatter, constitutes in many ways an ideal CPT laboratory. Using the large cross-sections (about $10^{9}$ barns) expected at a few keV $\bar{p}$ energy for the positronium reaction that can be written as
$\mathrm{Ps}+\overline{\mathrm{p}} \rightarrow \mathrm{e}^{-}+\overline{\mathrm{H}}\left(\mathrm{Ps}=\mathrm{e}^{+} \mathrm{e}^{-}\right)$, this collaboration estimates that about $1 \bar{H}$ atom per second can be formed from a sample of $10^{8} \bar{p}$ and has undertaken a pilot experiment on hydrogen formation by the charge conjugate reaction.

\section{Regions 3-4}

Once formed, $\overline{\mathrm{H}}$ atoms can only be confined by the interaction of their magnetic moment with an inhomogeneous magnetic field. The well depth obtained is determined by the field inhomogeneity, setting a practical limit on the energy of the $\overline{\mathrm{H}}$ which can be trapped. We must therefore turn to the 'cold' region 4 to study spectroscopy with trapped $\overline{\mathrm{H}}$ atoms, perhaps forming the $\overline{\mathrm{H}}$ here by recombination in cold $\mathrm{e}^{+} / \overline{\mathrm{p}}$ plasmas.

If the prospects for measuring such intriguing quantities as the $\overline{\mathrm{H}}$ Lamb shift by either of the above methods are at present rather distant, those offered in regions 3-4 for comparing the gravitational mass of the proton and the antiproton are much closer at hand. The assertion that antimatter falls under gravity in the same way as matter is not a result of CPT invariance but of the Galilean equivalence principle, a 'strong' form of which forms the basis of General Relativity (see "Gravity and Antimater", Scientific American, March 1988). GR essentially states that what really falls under gravity is energy and that all forms of energy fall (or 'weigh') equivalently. If energy alone weighs, gravitational fields cannot distinguish between matter and antimatter, since the rest energy contained in a proton and that contained in an antiproton are the same to the level of inertial mass equality. However, this extension of GR to the antimatter domain has never been verified empirically. There are theoretical grounds for believing that it may be necessary to relax the equivalence principle if successful quantization of gravity and its unification with strong and electroweak interactions at Big Bang energies is to be achieved.

One method for doing this measurement is experiment PS200 (Los Alamos/NASA Ames/TAMU/Colorado) in which a decelerating foil and confining field (as in PS196) will produce a $10 \mathrm{~K}$ sample of antiprotons. The antiprotons will be released $50-100$ at a time into a vertical flight tube and the time of flight spectrum over $1 \mathrm{~m}$ of their upward path against gravity will be measured. Antiprotons with less than a critical velocity will fall back down before they reach a detector placed at the upper level, resulting in a cutoff in the spectrum which depends on $g$. Since the gravitational potential energy change over $1 \mathrm{~m}$ is 0.1 $\mu \mathrm{V}$, only the extreme low energy tail of the $10 \mathrm{~K}$ (1 meV) Maxwell-Boltzmann distribution contributes information about $g$.

The proponents of the anticyclotron are studying an alternative method of measuring the effect of gravity on antiprotons. Suppose we take a coordinate system in which gravity acts along $y$ (the $x-z$ plane horizontal). The cyclotron orbit of an antiproton moving under gravity through a magnetic field $\boldsymbol{B}$ parallel to $z$ will drift in the direction $g \times B$ at a constant speed $u=m g \times B / e B^{2}$. This gravitational magnetron effect is very small $\left(1 \mathrm{~mm} / \mathrm{s}\right.$ for $\left.B=10^{-4} \mathrm{~T}\right)$, but the drift speed is independent of the antiproton's thermal velocity parallel to $B$. Measuring such a small velocity would not be easy but the method offers several attractive features. Yet another variant would be to try to measure gravity on (neutral) antihydrogen atoms, thereby avoiding many difficulties involved in screening out electromagnetic effects to the gravitational level.

We have thus arrived at a paradox within our initial paradox - that experiments on the coherent gravitational interaction between antiprotons at $\mu \mathrm{eV}$ energies and the $10^{51}$ or so nucleons in the earth may throw light on physics in the Planck energy region $\left(10^{19} \mathrm{GeV}\right)$ where strong, electroweak and gravitational interactions are expected to have the same strength. Such cosmic energies must remain forever beyond our reach by direct methods.

\section{REFERENCES}

[1] Andersen L.H. et al., Phys. Rev. A40 (1989) 7336.

[2] Bacher R. et al., Phys. Rev. A38 (1988) 4395.

[3] Eades J. et al., Int. School of Physics with Exotic Atoms, Erice (1989).

[4] Eades J. and Simons L.M., Nucl. Instr. Meth. A279 (1989) 368.

\section{Formal Announcement}

The Eighth General Meeting of the Members of EPS will be held in Amsterdam on Wednesday, 5 September 1990.

Applicants for membership awaiting acceptance will be admitted provided the first membership fee has been paid.

Members are invited to make proposals for topics to be placed on the Agenda in time for publication in the May issue of Europhysics News.

Preliminary Agenda

1. Report of the President

2. Report of the Secretary

3. Report of the Treasurer

4. Discussion of subjects of general interest according to suggestions and proposals put forward by Members. 